\title{
Can there be an elegant spin-orbital decomposition of the nucleon magnetic moment?
}

\author{
Xiang-Song Chen, ${ }^{1, *}$ Wei-Min Sun, ${ }^{2}$ Di Qing, ${ }^{3}$ and Hong-Shi Zong ${ }^{2,4}$ \\ ${ }^{1}$ Department of Physics, Sichuan University, Chengdu 610064, China \\ ${ }^{2}$ Department of Physics, Nanjing University, Nanjing 210093, China \\ ${ }^{3}$ CERN, CH-1211 Geneva 23, Switzerland \\ ${ }^{4}$ CCAST (World Laboratory), P.O. Box 8730, Beijing 100080, China
}

(Received 27 March 2006; published 7 July 2006)

\begin{abstract}
Recently, Mekhfi [Phys. Rev. D 72, 114014 (2005)] remarked that when studying spin-orbital separation of the nucleon magnetic moment with the Gordon decomposition, one should keep a timederivative term because the quark fields depend on time. We clarify that this term vanishes identically in a rigorous formulation of the nucleon magnetic moment, which then can be elegantly separated into a spin part related to quark tensor charge, and an orbital part related to quark convection angular momentum. In a quark model description of the nucleon, however, such a time-derivative term might contribute because it is hard to construct a true Hamiltonian eigenstate of relativistic interacting quarks.
\end{abstract}

DOI: 10.1103/PhysRevD.74.017901

PACS numbers: 12.39.Ki, 13.40.Em

In Ref [1], we used the Gordon decomposition to derive an elegant relation between magnetic moment and angular momentum for a relativistic system. This relation unambiguously separates the nucleon intrinsic magnetic moment into a spin part related to the quark tensor charge, and an orbital part related to the quark "convection" angular momentum. Recently, Mekfhi [2] remarked that our derivation erroneously omitted a time-derivative term. We supplement here why this term vanishes identically when one rigorously studies the intrinsic magnetic moment of a particle, either fundamental or composite. We also call attention that in a phenomenological model description of a composite particle this term might nevertheless be nonzero, because the model wave function may not be a true Hamiltonian eigenstate. In such a case one must be cautious at which magnetic moment formula to use.

Gordon decomposition separates the Dirac vector current $j^{\mu}=\bar{\psi} \gamma^{\mu} \psi$ into a convection part and a spin part:

$$
\bar{\psi} \gamma^{\mu} \psi=\frac{i}{2 m} \bar{\psi} \stackrel{\leftrightarrow}{D}^{\mu} \psi+\frac{1}{2 m} \partial_{\nu}\left(\bar{\psi} \sigma^{\mu \nu} \psi\right) \equiv j_{C}^{\mu}+j_{S}^{\mu},
$$

where $m$ is the mass of the Dirac field, $\stackrel{\leftrightarrow}{D}^{\mu}=D^{\mu}-\leftarrow_{D}^{\mu}$ is the covariant derivative. The Gordon decomposition follows directly from the equation of motion. In the case of free field, $D^{\mu}$ is replaced with $\partial^{\mu}$.

In [1], we remarked that the time-derivative term in Eq. (1) does not contribute to the nucleon magnetic moment. However, Mekhfi argues in [2] that this timederivative term cannot be thrown away because the quark fields depend on time in a nucleon. To clarify this issue, one must keep in mind that the intrinsic magnetic moment of a particle is defined in its Hamiltonian eigenstate with momentum close to zero [3]. In such states, a time-

*Electronic address: cxs@scu.edu.cn derivative term can be discarded identically: For any Heisenberg operator $\mathcal{O}$, we have the Heisenberg equation of motion $\partial_{t} \mathcal{O}=i[H, \mathcal{O}]$, where $H$ is the total Hamiltonian of the system. When taking expectation value in an eigenstate of $H, \partial_{t} \mathcal{O}$ vanishes for any operator $\mathcal{O}$.

The above nonperturbative conclusion can be verified perturbatively if one knows how to construct a Hamiltonian eigenstate of the particle. For example, one can easily check by straightforward calculation that at 1-loop order the time-derivative term in Eq. (1) does not contribute to the anomalous magnetic moment of the electron (despite that the electron field is nontrivially time-dependent when interacting with the photon field).

After dropping the time-derivative term in Eq. (1), we can elegantly decompose the magnetic moment operator $\vec{\mu}=\frac{1}{2} \int \mathrm{d}^{3} x \vec{r} \times \vec{j}$ into an orbital part and a spin part [1]:

$$
\begin{aligned}
\vec{\mu} & =\frac{1}{2 m} \int \mathrm{d}^{3} x \vec{r} \times \bar{\psi} \frac{1}{2 i} \stackrel{\leftrightarrow}{D} \psi+\frac{1}{2 m} \int \mathrm{d}^{3} x \bar{\psi} \vec{\Sigma} \psi \\
& \equiv \vec{\mu}_{L}+\vec{\mu}_{S} .
\end{aligned}
$$

The advantage of this expression is that the spin part is related to the quark tensor charge, which can be accessed experimentally and calculated reliably with lattice QCD [1]. However, one should not promptly employ this expression in a phenomenological quark model calculation. The dilemma is that it is hard to construct a true eigenstate of the total Hamiltonian of a relativistic interacting system, hence a time-derivative term $\partial_{t} \mathcal{O}=i[H, \mathcal{O}]$ might be nonzero in this model and Eq. (2) might be invalid.

This work was supported in part by China NSF (Grant No. 10475057 and No. 10575050). 
[1] X. S. Chen, D. Qing, W. M. Sun, H. S. Zong, and F. Wang, Phys. Rev. C 69, 045201 (2004).

[2] M. Mekhfi, Phys. Rev. D 72, 114014 (2005).
[3] S. Weinberg, The Quantum Theory of Fields (Cambridge, New York, 1995), Sec. 10.6. 